\title{
A Novel Effect of Acyclovir on Hair Growth in BALB/c Mice: A Promising Future for Finding a New Topical Drug for the Treatment of Hirsutism
}

This article was published in the following Dove Press journal: Clinical, Cosmetic and Investigational Dermatology

\author{
Soran Sameei ${ }^{1}$ \\ Hamid Soraya $^{2}$ \\ Morteza \\ Ghasemnejad-Berenji $\mathbb{D}^{2}$ \\ 'Student Research Committee, Urmia \\ University of Medical Sciences, Urmia, \\ Iran; ${ }^{2}$ Department of Pharmacology and \\ Toxicology, School of Pharmacy, Urmia \\ University of Medical Sciences, Urmia, \\ Iran
}

\begin{abstract}
Purpose: Hirsutism (ie, terminal hair growth on the face and body in a male-like pattern in women) is a common dermatological disorder in women, with psychosocial implications. Consequently, there is demand for finding novel pharmacological treatments and agents that can safely reduce hair growth. This study aimed to investigate the potential effect of topical acyclovir on hair growth in mice.
\end{abstract}

Methods: In this study, twenty-four female BALB/c mice were randomly divided into three groups in order to evaluate the hair growth-reducing effects of acyclovir (control group, vehicle group, and acyclovir group). Topical acyclovir 5\% was applied on the shaved denuded skin of mice. Topical application onto the backs of the animals was performed twice daily for 28 consecutive days. The time (in days) required for hair growth initiation as well as completion of hair growth in dorsal skin of animals were recorded. On day 28 , horizontally cut biopsy samples were removed and the numbers of hair follicles were counted, and the diameter of hair follicles was measured under high-field microscopy by a specialist blinded to the treatments.

Results: Hair growth initiation time was significantly increased with acyclovir, as compared to control and vehicle groups. The time required for complete hair growth in control and vehicle groups were $18 \pm 0.68$ and $19 \pm 1.41$ days, respectively; however, the hair growth completion in acyclovir-treated animals was not observed at the end of the experiment. Furthermore, the length of hairs in treatment group was significantly shorter than the control group at the end of the study $(\mathrm{P}<0.001)$. In histologic examination, the count and the diameter of hair follicles in deep subcutis were significantly decreased.

Conclusion: The results of this study, for the first time, showed that topical administration of acyclovir might have inhibitory effects on hair growth in experimental animals; however, further studies are required to understand its mechanism.

Keywords: hair growth, acyclovir, topical, mice, hair follicles, hirsutism

\section{Introduction}

Hirsutism is the presence of excess body or facial terminal (coarse) hair growth in females in a male-like pattern; this affects 5-15\% of women, and is an important sign of underlying androgen excess. ${ }^{1}$ Although excessive body or facial hair generally is not a life-threatening condition, its profound impact on social interactions and on patients' psychological wellbeing is undeniable. ${ }^{2}$ The available methods for treating this condition are electro-epilation, LASER (Light Amplification by the Stimulated Emission of Radiation) for hair removal, Intense Pulsed Light, Eflornithine, oral anti-androgens, oral contraceptives, and finasteride. ${ }^{3,4}$ These
Correspondence: Morteza GhasemnejadBerenji

Department of Pharmacology and

Toxicology, School of Pharmacy, Urmia

University of Medical Sciences, PO Box:

57I57993 I3, Urmia, Iran

$\mathrm{Tel} / \mathrm{Fax}+98$ 44-32754996

Email morteza.ghasemnejad@yahoo.com 
methods and hormonal therapies such as anti-androgenic agents also have transient effects and notable cost and side effects, therefore researchers are still seeking safer and more economical methods of treatment and finding new topical drugs with more efficacy, lower cost, and fewer side effects, can be valuable..$^{5-7}$ Acyclovir, an acyclic guanosine nucleoside analog, is an antiviral medication commonly prescribed for herpes simplex virus, cytomegalovirus, and varicella zoster virus (which manifests as chickenpox and shingles). The drug acts as a nucleotide analog and inhibits viral replication. ${ }^{8}$ According to our study on the effects of topical acyclovir on the skin of mice, we accidentally found that this drug potentially reduced hair growth. Previous studies have reported that hair loss is one of the uncommon side effects of oral acyclovir. ${ }^{9}$ However, no report has been issued on the effect of topical acyclovir on hair growth. Based on this possibility and our observations, we studied the effects of topical application of acyclovir on hair growth in mice.

\section{Methods and Materials Experimental Animals}

Female mice (6-8 weeks old, weighing $25-30$ g) were used for all the experimental procedures. The animals were purchased from Animal House of Urmia University of Medical Sciences. Animal experiments were conducted in accordance with the NIH guide for the care and use of laboratory animals. The animals were maintained under the standard laboratory conditions at $22 \pm 2^{\circ} \mathrm{C}$, relative humidity of $50 \pm 10 \%$ and normal photoperiod $(12 \mathrm{~h}$ dark/ $12 \mathrm{~h}$ light). Water and suitable nourishment were available for all mice in their cages throughout the study. The experiments were carried out following due approval of Urmia University of Medical Sciences Ethics Committee (IR.UMSU.REC.1398.175) which is in line with National Institutes of Health publication, 8th edition, and revised in 2011.

\section{Experimental Procedures}

Mice were randomly divided into three groups $(\mathrm{n}=8$ per group). The test groups included a control group (received no treatment), acyclovir group (treated with acyclovir 5\% cream), and vehicle group (treated with same cream formulation without acyclovir); all the animals were depilated topically with depilatory cream on dorsal skin. The commercially available 5\% acyclovir (parsdarou) was obtained from our local hospital pharmacy. Each gram of acyclovir cream 5\% contains $50 \mathrm{mg}$ of acyclovir and the following inactive ingredients: cetostearyl alcohol, poloxamer 407, propylene glycol, sodium lauryl sulfate (Sigma-Aldrich), mineral oil, water, and white petrolatum (Sepidaj, Tehran, Iran). The chemicals were pharmaceutical grade. The same formulation without acyclovir was used for vehicle group. This formulation was prepared in the pharmaceutical laboratory of faculty of pharmacy. The acyclovir cream and the same formulation without acyclovir respectively were applied to the denuded area of the treatment and vehicle groups twice a day and a control group received no treatment. This treatment was continued for 28 days.

\section{Hair Length Determination}

The hair regrowth at $7,14,21$, and 28 days after beginning of topical application was observed visually and recorded. ${ }^{10}$

\section{Histopathological Processing and Histomorphometry}

At the end of the experiment the mice were euthanized by overdose of pentobarbital. Dorsal skin measuring $15 \mathrm{~cm}^{2}$ was excised and stretched out on flat glass surface and was maintained in $4 \%$ formalin. $8 \mathrm{~mm}$ punch biopsies were excised from center of the skin, bisected, and embedded in paraffin blocks to obtain both longitudinal and transverse sections. $5 \mu \mathrm{M}$ sections were cut and stained with hematoxylin and eosin (H\&E). Digital photomicrographs were taken from representative areas at a fixed magnification of $\times 100$. All the images were cropped in a fixed area of 700 pixel width. From the sections, the number of hair follicles per millimeter of the skin, and the diameter of hair follicles were determined using high power microscope field and expressed as mean $\pm \mathrm{SD}$. $^{11}$

\section{Hair Weight Determination}

After euthanization, a $1 \mathrm{~cm}^{2}$ area of dorsal skin with hair and without hair was cut from all the mice of each group at the end of the study and weighed with the analytical balance. After measuring, hair weight was calculated by subtracting skin weight from skin with hair and without hair. $^{12}$

\section{Statistical Analysis}

All data were expressed as the mean \pm standard deviation (SD). All statistical analyses were done with GraphPad Prism 6. Statistical analysis for all the results was done using one- 
way analysis of variance (ANOVA) and post-comparison was carried out with Tukey's Test for post hoc analysis. A value of $\mathrm{P}<0.05$ was considered to be statistically significant.

\section{Results}

\section{In vivo Hair Growth Effect}

It was observed that the hair growth initiation and completion time from the shaved area was significantly increased upon treatment with topical acyclovir. Results are shown in Table 1 - in control and vehicle groups, hair growth was initiated in denuded area in the second week, whereas it was noted in the third week in acyclovir-treated group. A macroscopic view of hair regrowth at 7-day intervals for each group is presented in Figure 1. The results showed that mice treated with acyclovir had lower numbers of hairs in comparison to both control and vehicle groups. At the end of the 28th day, the acyclovir-treated mice all appeared healthy without any evidence of systemic toxicity or local side effects, such as swelling or redness.

\section{Measuring of Hair Length}

Results are shown in Table 2 - it was observed that the length of hair from the shaved area for acyclovir, control, and vehicle groups was $5.5 \pm 0.05 \mathrm{~mm}, 11 \pm 0.2 \mathrm{~mm}$, and 10 $\pm 0.1 \mathrm{~mm}$ respectively at the end of the experiment. Thus, acyclovir reduced the rate of hair growth.
Table I Effect of Acyclovir on Qualitative Hair Growth

\begin{tabular}{|l|l|l|}
\hline Groups & $\begin{array}{l}\text { Hair Growth } \\
\text { Initiation Time (Days) }\end{array}$ & $\begin{array}{l}\text { Hair Growth } \\
\text { Completion Time } \\
\text { (Days) }\end{array}$ \\
\hline $\begin{array}{l}\text { Control } \\
\text { Vehicle }\end{array}$ & $11 \pm 0.57$ & $18 \pm 0.68$ \\
$\begin{array}{l}\text { Acyclovir } \\
5 \%\end{array}$ & $17 \pm 0.45^{* * *}$, & $\begin{array}{l}19 \pm 1.41 \\
\text { Not complete at the end of } \\
\text { the 28th day }\end{array}$ \\
\hline
\end{tabular}

Note: Values are mean $\pm S D, \mathrm{n}=8$, *** $\mathrm{P}<0.001$, significance versus control, ${ }^{\# \#} \mathrm{P}<$ 0.001 , significance versus vehicle.

\section{Measuring of Hair Weight}

At the end of the course, the weight of newly grown hairs in a $1 \mathrm{~cm}^{2}$ area of dorsal skin of all test groups was measured and compared with each other. It was found that weight of hair was lowest for acyclovir-treated group. The weight of hair was measured to be $35 \mathrm{mg}$ / $\mathrm{cm}^{2}$ area of dorsal skin for acyclovir group, while it was found to be 50 and $57 \mathrm{mg} / \mathrm{cm}^{2}$ for control and vehicle groups respectively (Figure 2 ).

\section{Histologic Examination}

Longitudinal and transverse section of dorsal skin specimen of different treatment groups stained with hematoxylin and eosin (H\&E) is presented in Figure 3. In histologic examination, the hair follicle count in deep subcutis and the diameter of hair follicles were

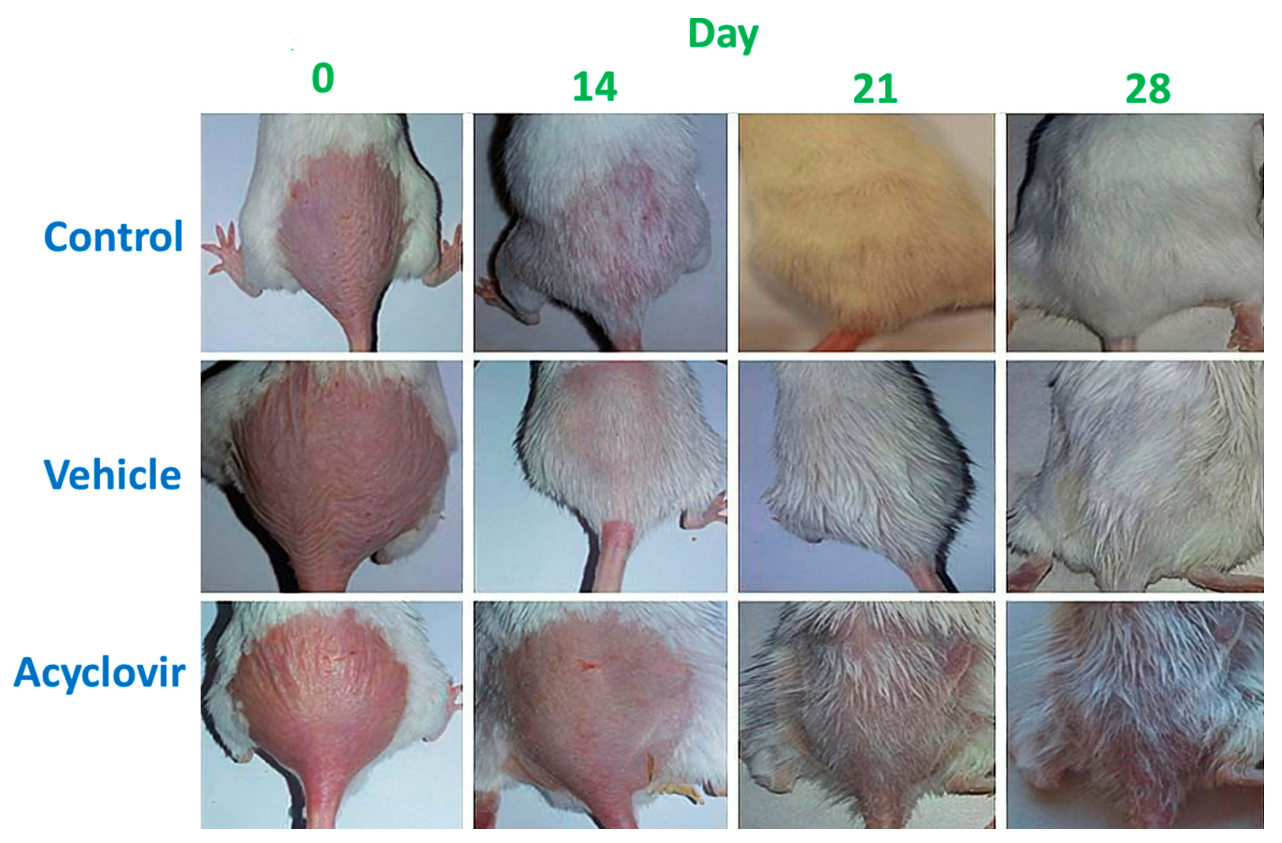

Figure I Effect of topical acyclovir on hair growth on different treatment days. BALB/c mice were shaved and received topically applied vehicle or acyclovir for 28 days, the control group did not receive anything ( $\mathrm{n}=\mathbf{8}$ for each group). Photographs were taken every week after applying acyclovir or vehicle on the shaved dorsal skin. Less white coloration was observed in acyclovir-treated mice than vehicle and control. 
Table 2 Effect of Acyclovir on Hair Length of Mice

\begin{tabular}{|l|l|l|l|}
\hline \multirow{2}{*}{ Group } & \multicolumn{3}{|l|}{ Length of Hair $(\mathbf{m m})$} \\
\cline { 2 - 4 } & Day I4 & Day 2 I & Day 28 \\
\hline Control group & $3.5 \pm 0.8$ & $7.4 \pm 1.2$ & $11 \pm 0.2$ \\
Vehicle group & $3.7 \pm 0.3$ & $6.9 \pm 0.8$ & $10 \pm 0.1$ \\
Acyclovir group & $1.1 \pm 08^{* * *}, \ldots \ldots$ & $3.7 \pm 0.6^{* * *}, \ldots \ldots$ & $5.5 \pm 0.05^{* * *}, \ldots \ldots$ \\
\hline
\end{tabular}

Note: Values are mean $\pm S D, n=8$, *** $\mathrm{P}<0.001$, significance versus control, \# $>0.001$, significance versus vehicle. ( $n=25$ hairs).

significantly decreased in acyclovir group as compared to control and vehicle groups. The number of hair follicles was found to be 4.33 hair follicles per $\mathrm{mm}$ of skin in acyclovir group. This was significantly $(\mathrm{P}<0.001)$ lower than for vehicle and control groups. The diameter of hair follicles was measured to be $30.83 \mu \mathrm{m}$ for acyclovir group, while it was found to be 49.50 and $47.50 \mu \mathrm{m}$ for control and vehicle groups respectively. The mean number of counted hair follicles and diameter of hair follicles in each group are reported in Figure 4.

\section{Discussion}

Hirsutism, a highly prevalent problem among women, may lead to psychological disorders, including anxiety, depression and low self-esteem; furthermore this condition can cause withdrawal from social interaction and isolation. ${ }^{13}$ The present study indicated that topical acyclovir was effective in decreasing hair growth without any notable side effects such as redness or swelling in normal mice. In

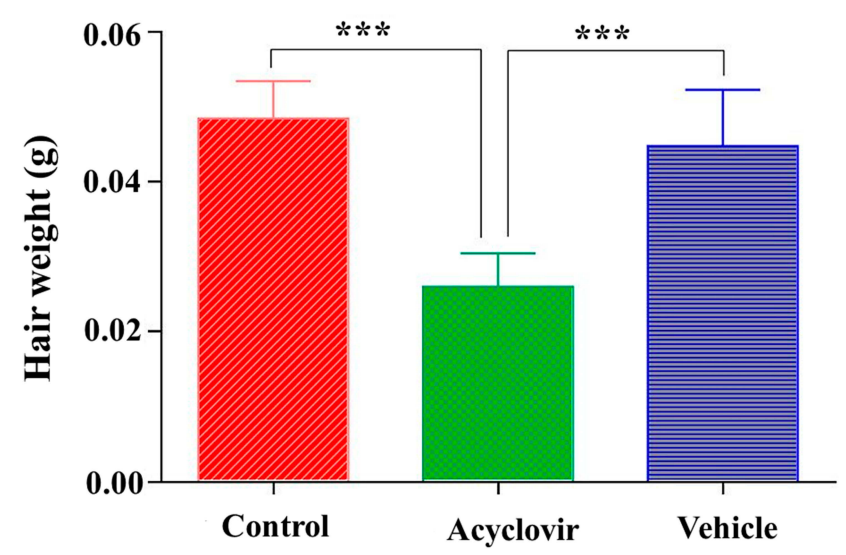

Figure 2 Weight of hair per $\mathrm{cm}^{2}$ area of dorsal skin after 28 days. Values are the mean \pm SD of eight mice. ${ }^{* * *} \mathrm{p}<0.001$ (one-way ANOVA, Tukey's test).

this study, the back skin of BALB/c mice was treated with daily topical application of acyclovir for 28 days. In normal control mice, faint hair regrowth was observed at 14 days and full regrowth was observed at 28 days after depilation. However, in the acyclovir group, only faint hair regrowth was observed at 21 days and sparse regrowth was observed at 28 days after depilation. To further investigate the hair growth inhibition, we assessed the effect of acyclovir on the density of hair follicles by staining with H\&E. Consistently, topical application of acyclovir substantially decreased the number of hair follicles as compared to the control group. Furthermore, according to our results, the diameter of hair follicles was significantly decreased in treatment group when compared

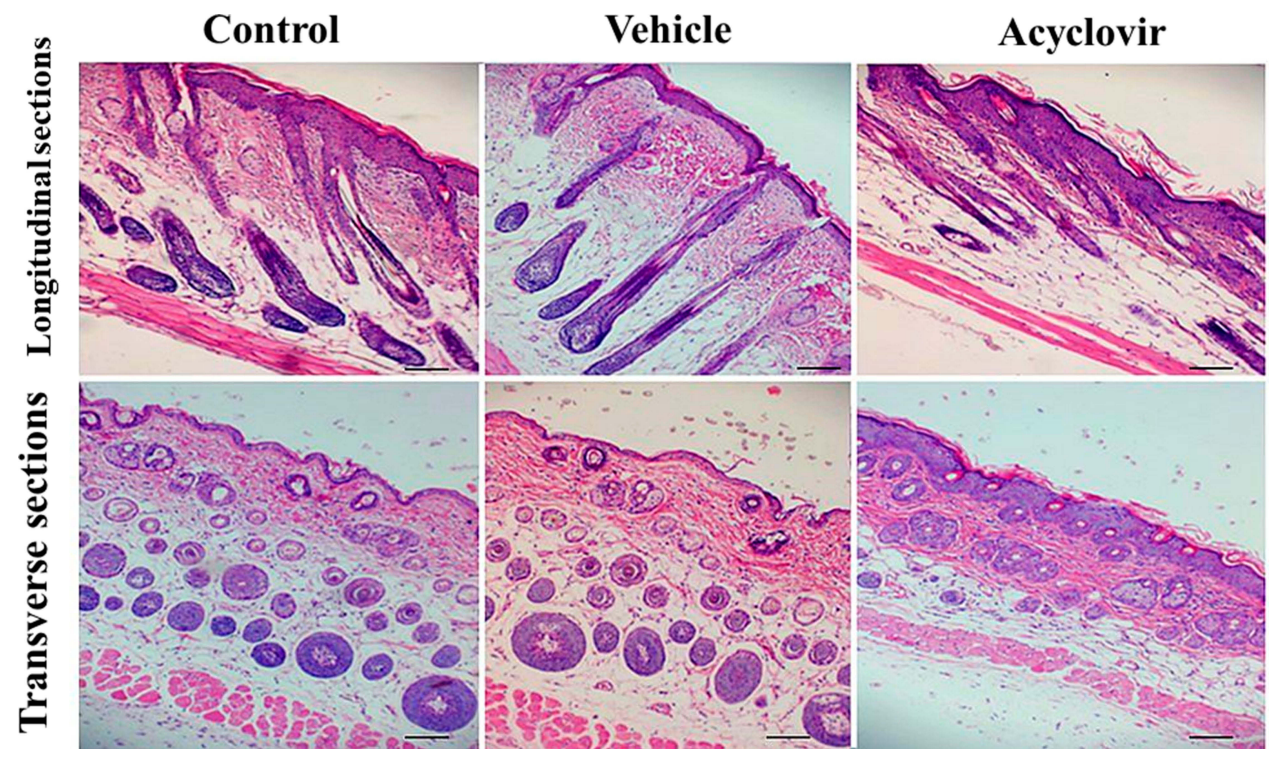

Figure 3 Longitudinal and transverse sections of dorsal skin specimens of different treatment groups stained with H\&E at the end of the 28 th day. The number of hair follicles in deep subcutis can be observed. The image shown is the representative picture of 8 mice. Bars: $100 \mu$ m. Topical acyclovir decreased the number of hair follicles. 

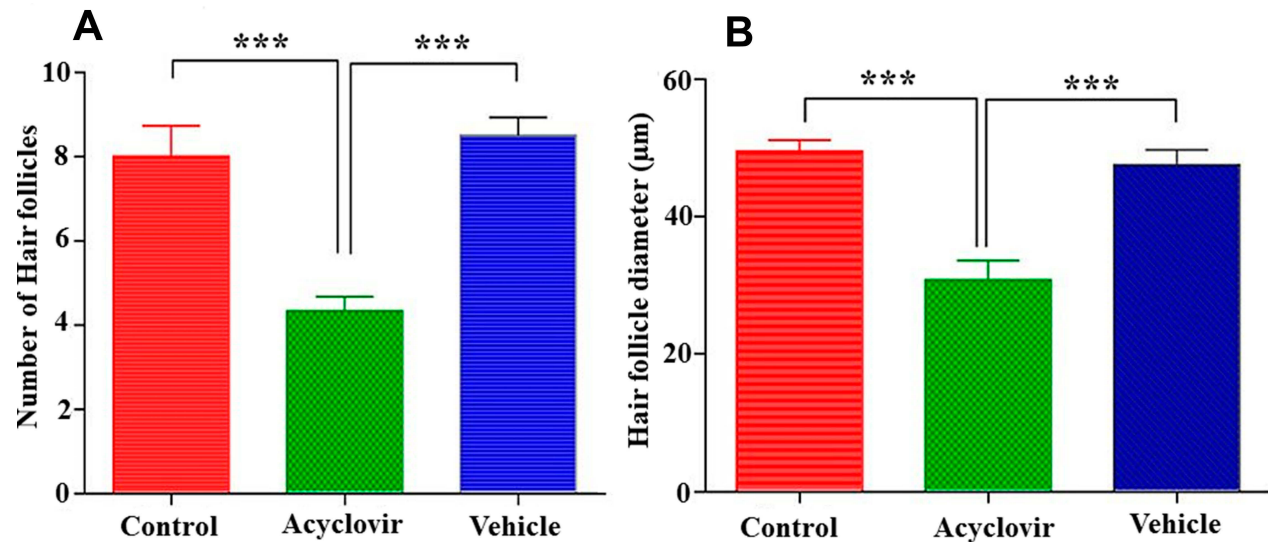

Figure 4 Number $(\mathbf{A})$ and diameter $(\mathbf{B})$ of hair follicles in deep subcutis. Values are the mean \pm SD of eight mice. ${ }^{* * *}$ p $<0.00$ I (one-way ANOVA, Tukey's test).

with the control group. Acyclovir is an antiviral medication. It is primarily used for the treatment of herpes simplex virus infections, chickenpox, and shingles. The current investigation was a preliminary study designed to determine the effects of topical acyclovir on hair growth. For the first time we reported the inhibitory effect of acyclovir on hair growth in mice. This effect may be caused by the fact that acyclovir is a synthetic analog of the purine nucleoside, guanosine, so it may induce abrupt cessation of mitotic activity in rapidly dividing hair matrix cells (anagen effluvium) or may bring the hair follicle to premature rest (telogen effluvium). However, further studies should be directed in order to understand the exact mechanism of this effect. The drug-induced alopecia by oral acyclovir has been reported previously by Sharma et al. They revealed that alopecia may be considered as a possible complication following oral acyclovir. They concluded that acyclovir should be discontinued in case alopecia occurs. ${ }^{9}$ Our study is in line with this case report. We observed the significant inhibitory effect of topical acyclovir on hair growth. We propose that acyclovir plays an important role as an inhibitor of hair follicle growth and may function as an additional therapeutic agent for hirsutism. Unwanted body hair can be emotionally and socially devastating, ${ }^{14}$ resulting in the search for various treatment modalities. The simplest and most popular method is the use of depilatory materials, which chemically remove hair from the skin surface by dissolving keratin but only has a temporary effect. Unfortunately, there are some side defects, such us skin allergy, paradoxical hypertrichosis or skin burn. ${ }^{15}$ According to the results of this present study, acyclovir can decrease the density of hair follicles with unknown mechanism, and can be a potential hair removal drug. The discovery of novel economic and safe methods for treatment of hirsutism is of great importance as the notable cost of routinemethods such as electrolysis and photo-epilation and serious side effects of oral hormonal therapies such as anti-androgenic agents. ${ }^{16-18}$ In our study, side effects such as local inflammation, edema, or scaling of skin from topical acyclovir were not observed, therefore this drug can be suggested as a new topical agent for decreasing hair growth in hirsutism. However, further studies should be performed to confirm this hypothesis and additional clinical research is required to further determine the potential of acyclovir as a pharmacologic strategy for hirsutism.

\section{Acknowledgment}

This research was supported by Urmia University of Medical Sciences (Grant Number: 2576).

\section{Disclosure}

No potential conflict of interest was reported by the authors.

\section{References}

1. Yildiz BO, Bolour S, Woods K, Moore A, Azziz R. Visually scoring hirsutism. Hum Reprod Update. 2009;16(1):51-64. doi:10.1093/ humupd/dmp024

2. Rahnama Z, Sohbati S, Safizadeh H. Effect of hirsutism on quality of life: a study in Iranian women. J Pak Assoc Dermatol. 2016;23 (1):28-33.

3. Alijanpour R. The effect of topical finasteride $0.5 \%$ on the outcome of diode laser therapy in the treatment of excess facial hairs in the women with hirsutism. J Pak Med Assoc. 2016;66:1107-1110.

4. Ezeh U, Huang A, Landay M, Azziz R. Long-term response of hirsutism and other hyperandrogenic symptoms to combination therapy in polycystic ovary syndrome. J Women Health. 2018;27(7):892-902. doi:10.1089/jwh.2017.6833 
5. Blume-Peytavi U, Hahn S. Medical treatment of hirsutism. Dermatol Ther. 2008;21(5):329-339. doi:10.1111/j.1529-8019.20 08.00215.x

6. Brodell LA, Mercurio MG. Hirsutism: diagnosis and management. Gend Med. 2010;7(2):79-87. doi:10.1016/j.genm.2010.04.002

7. Chang D. Hair loss and hirsutism management. Cosmetol Oro Facial Surg. 2017;3(120):2.

8. Kłysik K, Pietraszek A, Karewicz A, Nowakowska M. Acyclovir in the treatment of herpes viruses-a review. Curr Med Chem. 2019.

9. Sharma A, Mohan K, Sharma R, Nirankari VS. Alopecia following oral acyclovir for the treatment of herpes simplex keratitis. Middle East Afr J Ophthalmol. 2014;21(1):95. doi:10.4103/0974-9233.12 4131

10. Adhirajan N, Kumar TR, Shanmugasundaram N, Babu M. In vivo and in vitro evaluation of hair growth potential of Hibiscus rosa-sinensis Linn. $J$ Ethnopharmacol. 2003;88(2-3):235-239. doi:10.1016/S0378-8741(03)00231-9

11. Park H-J, Zhang N, Park DK. Topical application of Polygonum multiflorum extract induces hair growth of resting hair follicles through upregulating Shh and $\beta$-catenin expression in C57BL/6 mice. J Ethnopharmacol. 2011;135(2):369-375. doi:10.1016/j.jep.2011.03.028
12. Yoon JI, Al-Reza SM, Kang SC. Hair growth promoting effect of Zizyphus jujuba essential oil. Food Chem Toxicol. 2010;48 (5):1350-1354. doi:10.1016/j.fct.2010.02.036

13. Baig T, Aman S, Nadeem M, Kazmi AH. Quality of life in patients of hirsutism. J Pak Assoc Dermatol. 2016;24(3):217-223.

14. Blume-Peytavi U. An overview of unwanted female hair. $\mathrm{Br}$ J Dermatol. 2011;165:19-23. doi:10.1111/j.1365-2133.2011.10632.x

15. Zoumaras J, Kwei J-S-S, Vandervord J. A case review of patients presenting to Royal North Shore Hospital, with hair removal wax burns between January and November 2006. Burns. 2008;34 (2):254-256. doi:10.1016/j.burns.2007.03.018

16. Anjum MU, Yasmin S, Riaz H, Shah SH. Hirsutism. Prof Med J. 2016;23(06):741-745.

17. Eide K, Adam P. Is spironolactone better than finasteride for symptoms of hirsutism in transgender women? Evidence-Based Pract. 2019;22(8):11-12.

18. Subha R, Suganthy V, Tharini G. Intense pulsed light for the treatment of hirsutism. Int J Res. 2018;4(2):219.

\section{Publish your work in this journal}

Clinical, Cosmetic and Investigational Dermatology is an international, peer-reviewed, open access, online journal that focuses on the latest clinical and experimental research in all aspects of skin disease and cosmetic interventions. This journal is indexed on CAS.
The manuscript management system is completely online and includes a very quick and fair peer-review system, which is all easy to use. Visit http://www.dovepress.com/testimonials.php to read real quotes from published authors. 